\title{
Chunking processes in the learning of event sequences: Electrophysiological indicators
}

\author{
FRIEDERIKE SCHLAGHECKEN \\ Birkbeck College, London, England \\ BIRGIT STÜRMER \\ Humboldt-Universität, Berlin, Germany \\ and \\ MARTIN EIMER \\ Birkbeck College, London, England
}

\begin{abstract}
The present study investigated whether effects of implicit learning (IL) are due to well-learned and explicitly represented parts of the stimulus material ("chunks"). To this purpose, event-related brain potentials (ERPs) were recorded during an oddball-version of a serial reaction time (RT) task: At unpredictable positions within a 16-item letter sequence, single deviant items replaced an item of the repeatedly presented standard sequence. After acquisition, the "process dissociation procedure" (Jacoby, 1991) was adopted to identify explicitly learned sequence parts for each participant. Acquisition of sequence knowledge was reflected in faster RTs for standard items than for deviant items and in enhanced N2b and P3b components for deviant items. While the ERP effects were obtained for explicitly represented sequence parts only, RT effects were independent of subsequent reproduction performance. These results indicated that (1) ERPs are a valid measure of explicit knowledge, (2) implicit and explicit knowledge coexist in serial RT tasks, and (3) chunking processes play a major role in the acquisition of explicit knowledge about event sequences.
\end{abstract}

In recent years, the notion of implicit learning (IL) has become well established in the literature on memory and learning. In a variety of tasks, participants show the ability to utilize the structure that underlies stimulus presentation but are unable to report any kind of regularity in a subsequent test of their explicit knowledge. Therefore, it is often concluded that a special type of learning exists, occurring without intention to learn and without concurrent awareness of the underlying regularity (see Reber, 1989, and Seger, 1994, for reviews). However, the question of how this type of learning is accomplished is far from settled. Numerous researchers claim that explicit and implicit learning are the outcomes of two fundamentally different functional systems (e.g., Schacter, 1994; Squire, 1994), whereas other authors argue that explicit and implicit learning are more likely task-specific varieties of one unitary learning mechanism (e.g., Perruchet, 1994; Roediger, 1990a, 1990b; Shanks \& St. John, 1994).

The claim that IL is a special form of learning mainly relies on the assumption that it occurs in the absence of conscious knowledge about the structure of the to-belearned material. Thus, in order to argue in favor of IL as a special form of learning, it is critical to prove the absence

The present experiment was conducted at the Department of Psychology, Ludwig-Maximilians-University, Munich, Germany. Correspondence should be addressed to F. Schlaghecken, Department of Experimental Psychology, Birkbeck College, Malet Street, London WCIE 7HX, England (e-mail: f.schlaghecken@bbk.ac.uk). of conscious awareness of the underlying regularity during acquisition. To this purpose, experiments on IL usually consist of two parts: a training phase, during which the acquisition of knowledge regarding the structure of the presented material can take place, and a test phase, designed to find out how much of this knowledge was acquired explicitly.

A typical paradigm in the study of IL is the serial reaction time (RT) task introduced by Nissen and Bullemer (1987). On each trial, a stimulus is presented in one of four possible locations, and participants have to press one of four response buttons corresponding to the location of each stimulus. In the experimental condition, the sequence of stimulus locations follows a repeating pattern. After prolonged training, the regular sequence is switched to a random sequence. Participants are neither told about the existence of a regularity nor about the switch from structured to random presentation. In a control condition, participants are confronted with random sequences only. Finally, the explicit knowledge acquired by the experimental group is tested by asking the participants to verbally reproduce the stimulus sequence and/or to perform a prediction test of the sequence (i.e., indicating which position would be the next after a small part of the sequence has been presented). Typical results of an experiment like this are (1) RTs decrease faster during training when participants are confronted with a structured sequence than when they are confronted with a random sequence, (2) shifting to a random sequence after several blocks of 
structured presentation leads to a marked increase in RTs, and (3) these effects can be observed even for participants who perform poorly in tests of explicit knowledge (e.g., Cohen, Ivry, \& Keele, 1990; Curran \& Keele, 1993; Hartman, Knopman, \& Nissen, 1989; Lewicki, Hill, \& Bizot, 1988; Reed \& Johnson, 1994; Stadler, 1989, 1993; Willingham, Greeley, \& Bardone, 1993; Willingham, Nissen, \& Bullemer, 1989).

This last finding is crucial for the notion of IL: The assumption that IL is a special form of learning is based mainly on the dissociation between performance increments due to regularities in stimulus presentation and the subsequent inability to consciously express this regularity in a recognition or prediction task. It is argued that (1) if participants show improved performance in the serial RT task, then they must have acquired some knowledge about the underlying regularities, (2) if they are not able to consciously express these regularities, then this knowledge can be regarded as being implicit, and (3) if the knowledge is implicit, then the learning was also implicit. Additional evidence supporting this hypothesis stems from neuropsychological studies showing that sequence learning is spared in amnestic patients who show severe impairments of conscious episodic memory (Nissen \& Bullemer, 1987; Nissen, Willingham, \& Hartman, 1989). This supports the conclusion that procedural knowledge about sequential structures can be acquired incidentally and in the absence of conscious knowledge about the sequence.

On the other hand, there has been some indication that explicit knowledge may play a more prominent role in sequence learning than was previously thought (e.g., Perruchet, 1994; Perruchet \& Amorim, 1992; Shanks \& St. John, 1994). Perruchet and Amorim (1992) used a recognition task in order to better assess the amount of explicit knowledge acquired during training. Participants had to decide whether or not four-item sequences were part of the previously presented sequence. Additionally, a reproduction task was used, in which participants were instructed to reproduce the previously delivered sequence using the same keys as before. Since there were marked correlations between RT benefits and both recognition and reproduction of sequence parts, the authors concluded that performance effects in the training period may be mediated not by implicit learning but by the conscious acquisition of explicit knowledge about small chunks of successive elements.

\section{The Problem of Identifying Implicit and Explicit Knowledge}

One problem for all conclusions regarding the nature of IL is that they rely on backward inferences from memory tests-performed after the acquisition period-to learning processes that took place during the preceding serial RT task. With this method, the amount of explicit knowledge may be underestimated, as argued by Perruchet and Amorim (1992). On the other hand, it seems also possible that the amount of explicit knowledge may be overestimated: One could argue, for example, that performance in subsequent tests of explicit knowledge may benefit from implicit knowledge.

To circumvent these problems, Jacoby (1991) recommended the use of a "process dissociation procedure" in order to separate explicit from implicit knowledge. Here, the same memory task has to be performed twice under opposite instructions. The logic underlying this procedure is as follows: It is assumed that, under the instruction to reproduce the material acquired before, both explicit and implicit knowledge will influence performance. Under the opposite instruction - namely, to avoid reproducing this material - still some parts of the previously learned material are likely to be reproduced, especially those parts that cannot be consciously suppressed because they are only implicitly (i.e., not consciously) known. By comparing performance under both instruction conditions, one thus may separate explicit from implicit knowledge. Although the process dissociation procedure has been widely criticized and several alternative models have been suggested (e.g., Gardiner \& Java, 1993; Joordans \& Merikle, 1993; see Reingold \& Toth, 1996, for an overview), the discussion usually only touches the problem of estimating the proportion of implicit influences on performance, but not the assumption that the method adequately identifies explicit influences on performance.

However, even if this method would reliably identify both explicit and implicit knowledge, the problem of the validity of backward inferences would not necessarily be solved: It seems still questionable whether explicit knowledge is solely due to explicit learning and whether implicit knowledge is solely due to implicit learning. In principle, there are two ways in which explicit versus implicit knowledge and explicit versus implicit learning may dissociate: First, one could imagine that, although learning was implicit, during recall (or shortly before), the knowledge acquired so far became consciously available (i.e., explicit). On the other hand, it seems possible that fragmentary explicit knowledge available during acquisition may have been forgotten when a memory test is administered afterwards (Shanks, Green, \& Kolodny, 1994; Shanks \& St. John, 1994). Trying to circumvent this problem by probing participants' conscious knowledge at different times during sequence learning has the unavoidable side effect of directing their attention to the inherent structure of the sequence and thereby probably altering their subsequent strategy in the serial RT task (Shanks et al., 1994). Thus, a nonreactive on-line measure of the development of conscious knowledge that does not depend on the participants' overt responding and can be obtained continuously while performing the serial RT task would be helpful.

\section{ERPs in IL Experiments}

In two previous studies (Baldwin \& Kutas, 1997; Eimer, Goschke, Schlaghecken, \& Stürmer, 1996), event-related brain potentials (ERPs) were used as an on-line measure for knowledge acquisition. In the Baldwin and Kutas 
(1997) study, IL was investigated in the context of a serial RT task, in which stimulus presentation (i.e., the movements of a visual stimulus on the computer screen) followed the rules of an underlying artificial grammar. On some trials, the stimulus would make a movement that violated the underlying grammar. ERP responses to these "ungrammatical" movements were compared with responses to "grammatical" movements. It was found that on trials in which no response was required ("standard" trials), around $300 \mathrm{msec}$ after stimulus onset ERPs elicited by ungrammatical movements exhibited a slightly more positive-going shift than ERPs elicited by grammatical movements. On trials in which a response was required ("target" trials), on the other hand, ERPs elicited by ungrammatical movements were more negative than ERPs elicited by grammatical movements in a latency range of $200 \mathrm{msec}-500 \mathrm{msec}$ after stimulus onset. Further analysis revealed that this effect was largely due to a delayed P3b component (a positive-going ERP deflection starting around 300 msec after stimulus onset) elicited by ungrammatical targets.

In the Eimer et al. (1996) study, the standard serial RT task was combined with a variation of the oddball paradigm. In the typical oddball paradigm, unexpected events ("deviants") are infrequently interspersed in a continuous stream of standard items. In the Eimer et al. study, stimulus deviance was defined with respect to the relative position of an item within a complex 10 -item letter sequence. Typically, deviant items in an oddball situation elicit an enlarged P3b component that is often preceded by an enhanced N2b (a negative-going deflection starting around $200 \mathrm{msec}$ after stimulus onset; see Donchin, 1981, Ritter et al., 1984, Ruchkin, Sutton, \& Tueting, 1975, Squires, Donchin, Herning, \& McCarthy, 1977, and Squires, Wickens, Squires, \& Donchin, 1976, for reviews). This ERP pattern was also obtained in the Eimer et al. study, even though participants were not informed about the presence of the sequence. Moreover, the N2b effect was large for participants showing substantial explicit knowledge of the sequential structure, smaller for participants with less explicit knowledge, and absent for participants showing no explicit knowledge at all.

An additional finding of the Eimer et al. (1996) study was that a significantly enlarged $\mathrm{P} 3 \mathrm{~b}$ was elicited by deviant items only under conditions in which learning was difficult (because the sequence was frequently interrupted by deviant items; Experiment 2), whereas under conditions in which sequence learning was easier (because deviant items occurred only half as often; Experiment 1), no such effect was present. Since the P $3 b$ effect is usually associated with the detection of improbable surprising events (e.g., Donchin, 1981, and Picton \& Hillyard, 1988 , for overviews and discussions), this pattern of results seems to be the reverse of what one should expect: Normally, one would assume that deviant items are more easily detected - and correspondingly elicit a larger $\mathrm{P} 3 \mathrm{~b}$ under an easy-learning condition than under a difficult- learning condition. There is, however, a post hoc explanation along the line of Perruchet's and Amorim's (1992) reasoning that may account for these results: In the "easy" condition, participants may have acquired information about the global structure of the stimulus presentation, including the knowledge that sometimes deviant items occurred. In the "difficult" condition, only chunks of a few successive elements may have been learned, so that deviant items replacing one of the chunk elements were found to be very surprising. Unfortunately, it was not possible to determine which chunks, if any, had been acquired in the Eimer et al. study. Thus, the assumptions regarding the unexpected $\mathrm{P} 3 \mathrm{~b}$ difference between the two experiments had to remain purely speculative. The present experiment was designed to test more directly the hypothesis that ERP effects of sequence learning are at least partly due to well-learned and explicitly represented chunks.

\section{EXPERIMENT \\ Evidence of Chunk Learning in a Serial RT Task}

In the present experiment, we pursued two main purposes. First, chunk learning should be encouraged. Therefore, sequence learning was made more difficult than in the Eimer et al. (1996) study. We assumed that a more complex sequence would prevent participants from acquiring knowledge about its global structure and would enforce learning of sequence parts. Consequently, sequence length was enlarged from 10 items to 16 items per sequence and a more arbitrary stimulus-response mapping was employed than in the previous experiments.

The second aim was to obtain a more valid measure of explicit knowledge in order to classify explicitly learned chunks for each participant individually. To attain such a measure, the process dissociation procedure of Jacoby (1991) was adopted. In the "inclusion" condition, participants were required to make positive use of the explicit knowledge acquired previously - that is, to reproduce as many items of the learned sequence as possible. In the "exclusion" condition, participants were asked to make negative use of the learned material - that is, to produce random sequences that did not resemble the sequence presented before. Assuming that participants follow the instructions, mainly items that had been learned but could not be remembered consciously (and, therefore, could not be suppressed consciously) should be reproduced in the exclusion condition. Consequently, an above-chance production of regular items in the exclusion condition could be ascribed to influences of implicit memory processes. Thus, by comparing task performance under both instructions, chunks of explicit knowledge could be identified as sequence parts that were reproduced (1) frequently and (2) predominantly in the inclusion condition. Since there is as yet no unequivocal criterion to decide how to calculate the proportion of implicit processing (see above), in the present experiment 
we focused solely on electrophysiological correlates of explicit knowledge. That is, although it was assumed that chunks identified on the basis of the process dissociation procedure did in fact represent explicit knowledge, no assumption was made as to whether performance at sequence parts that were not identified as chunks was influenced by implicit and/or explicit knowledge.

If the hypothesis that sequence learning is at least partly mediated by explicit chunk learning is correct, then the following pattern of results is to be expected: As in the Eimer et al. (1996) study, deviant items should elicit larger N2b and P3b deflections than should standard items. However, these effects should be restricted to elements of the sequence that form explicitly represented chunks ("chunk-internal" positions). For the remaining elements ("chunk-external" positions), no such effects should occur. Such a finding would provide conclusive evidence for the assumption that ERPs can be regarded as indicating consciously available explicit knowledge about a stimulus sequence. Moreover, since the serial RT task was not interrupted by any explicit memory retrieval task, this finding would support the notion that ERPs can be employed to monitor the "natural" development of explicit knowledge (i.e., knowledge that has not been induced by additional task requirements).

However, one potential confounding factor has to be taken into account. Baldwin and Kutas (1997, Experiment 1) showed that the difference between ungrammatical ERPs and grammatical ERPs on target trials was strongly related to RT on grammatical trials: On trials in which RT was fast (i.e., faster than on ungrammatical trials), substantial ERP effects occurred, whereas on trials in which RT was slow (i.e., as slow as on ungrammatical trials), the respective ERP waveforms were almost identical to those elicited on ungrammatical trials, both exhibiting a N2b and a late P3b. A similar association between ERP effects and RT effects was observed in the Eimer et al. (1996) study, in which ERP effects and RT effects were largest for participants who had acquired substantial explicit knowledge about the sequence structure, and ERP and RT effects were smallest for participants who had acquired almost no explicit knowledge. Thus, one may suspect that the $\mathrm{N} 2 \mathrm{~b}$ and P3b effects are not so much indicators of processing consciously detected deviants but result from a delay in onset latency of the response-related late positive shift. However, the Eimer et al. study put this issue to test by comparing ERPs elicited in two blocks of pseudo-random presentation with ERPs elicited by the same items in the standard sequence. Although RTs in random blocks and RTs to deviant items in sequence blocks were similarly delayed relative to $R T$ s to standard sequence items $(55 \mathrm{msec}$ on average), only deviant items in sequence blocks gave rise to distinct ERP waveforms, whereas ERPs elicited by random items did not differ from ERPs elicited by standard sequence items. Obviously, though, the existence of RT differences in a serial RT task is not sufficient to elicit the observed ERP effects; rather, these effects also require some processing of stimulus deviance.

\section{Method}

\section{Participants}

Fifteen paid volunteers ( 7 male, 8 female) 20-35 years of age (mean age $=25.7$ years) participated in the experiment. All participants were right-handed and had normal or corrected-to-normal vision.

\section{Stimuli and Apparatus}

The participants were seated in a dimly lit, electrically shielded, and sound-attenuated chamber, with four response buttons, one under each left and right index and middle finger. A computer screen was placed $100 \mathrm{~cm}$ in front of the participant's eyes, carefully positioned so that the stimuli occurred on the horizontal straightahead line of sight. Stimuli were uppercase letters D, H, R, and $K$ that were presented white-on-gray in the center of the screen, covering a visual angle of approximately $1^{\circ}$. Each stimulus remained on the screen until a response had been executed. Response latencies were recorded for each trial. The interval between the response and the presentation of the next stimulus was $200 \mathrm{msec}$.

\section{Procedure}

Serial RT task. The participants were asked to respond to the letter stimuli by pressing the appropriate response button as fast and correct as possible. Responses were mapped to the letters as follows: Letters $D$ and $H$ required a response with the left middle and index finger, respectively, and letters $R$ and $K$ required a response with the right index and middle finger, respectively. Thus, the stimulusresponse mapping was noncongruent, in that for left-hand responses, the letter appearing earlier in the alphabet required a response with the left finger, and the letter appearing later in the alphabet required a response with the right finger, whereas for right-hand responses, the reverse was true. The presence or absence of a sequence was not mentioned.

The experiment consisted of one training block, containing 100 trials, 18 experimental blocks of 256 trials each, and two blocks of the reproduction task, each with 160 trials. Successive blocks were separated by short rest periods, and a longer rest period was given after Experimental Block 9. In the training block, stimuli were presented in a pseudo-random order, with no immediate repetition of a single letter. If a participant found it particularly hard to learn the appropriate stimulus-response mapping, the training block was repeated (this was necessary for only 1 participant). In the experimental blocks, stimuli were presented in a particular 16-trial sequence ( $\mathrm{K}-\mathrm{D}-\mathrm{R}-\mathrm{K}-\mathrm{D}-\mathrm{H}-\mathrm{K}-\mathrm{R}-\mathrm{K}-\mathrm{H}-\mathrm{D}-\mathrm{R}-\mathrm{H}-\mathrm{D}-\mathrm{H}-\mathrm{R})$. Each block consisted of 16 repetitions of this sequence. The end of each sequence and the beginning of the next sequence were not marked, and each block could start at any position within the sequence. Given this sequence, left-hand and right-hand responses were equiprobable. The participants had to change the respond hand on successive trials as often as they had to respond with the same hand on 2 consecutive trials (eight times per sequence each). In each sequence, one standard stimulus was replaced by one stimulus letter that normally did not occur at that particular position within the sequence (deviant stimulus). Deviant letters were not identical with the immediately preceding letter or with the immediately following letter. Thus, throughout the whole experiment, identical stimuli never occurred on successive trials. After presenting a deviant stimulus, the sequence was continued with the next standard stimulus.

Memory tasks. At the end of the first experimental half, the participants were asked whether they had noticed anything special regarding the experimental circumstances. When the presence of a sequence was reported, the participants were asked to verbally 
reproduce this sequence. However, the existence of a sequence was neither confirmed nor denied. After finishing the experimental blocks, the participants were again asked whether anything particular had been noticed and were asked to reproduce verbally any repeating patterns they could remember.

Only after this procedure were the participants told about the existence of the sequence, but not about its structure. They were then instructed to perform two blocks of the reproduction task with 160 trials each. The participants had to press the same response buttons as during the serial RT task. With each buttonpress, the letter mapped onto the respective button appeared on the screen. One of the blocks required the reproduction of the parts of the sequence that the participant was able to remember (inclusion condition). In case the participant did not remember any regularity, he/she was encouraged to reproduce series that "seemed familiar" to him/her. In contrast, the exclusion condition required the participants to suppress the reproduction of explicitly remembered sequence parts. Therefore, the participants were instructed to produce random series of keypresses and to avoid reproducing series that appeared during the preceding serial RT task. Moreover, it was stressed that they should try to produce "real" random series of the four stimuli, avoiding immediate repetitions or simple rhythmical patterns. The participants were instructed to perform the reproduction tasks in a comfortable pace-ideally, the same pace they obtained during the experimental blocks - and neither to hurry nor to wait too long before each keypress.

Finally, the correct stimulus sequence together with five new stimulus sequences of 16 items each was presented on a sheet of paper. The participants were told that one of those sequences had been repeated 288 times during the serial RT task, only sometimes interrupted by a single item that violated the underlying rule. Additionally, they were informed that each block started at a different position of the sequence, so that the beginning and the end of each sequence was not marked. After debriefing, the participants were asked to identify the correct sequence. They were told that it could be difficult to remember the complete sequence and that, in case they did not recognize it, they should pick the sequence that seemed most familiar to them. They were, however, not encouraged to focus on parts of the sequence.

\section{EEG recording}

EEG was recorded with $\mathrm{Ag}$ - $\mathrm{AgCl}$ electrodes $(5 \mathrm{~mm}$ in diameter) from frontopolar, frontal, central, and parietal midline scalp sites ( $\mathrm{FPz}, \mathrm{Fz}, \mathrm{Cz}, \mathrm{Pz}$, according to the International 10-20 system), as well as from left and right central scalp sites $\left(\mathrm{C}^{\prime}\right.$ and $\mathrm{C}^{\prime}$, located $1 \mathrm{~cm}$ in front of $\mathrm{C} 3$ and $\mathrm{C} 4$, respectively). All electrodes were referenced to the right earlobe. Electrode impedance was kept below $5 \mathrm{k} \Omega$. The amplifier bandpass was $0.10-70 \mathrm{~Hz}$. EEG was sampled online every $7 \mathrm{msec}$

\section{Data Analysis}

Items at chunk-internal positions were identified for each participant individually on the basis of the reproduction tasks. Sequences of three or more successive letters that (1) were part of the experimental sequence, $(2)$ were produced with a higher than chance frequency in the inclusion block, and (3) occurred in the inclusion block more often than in the exclusion block (at least three occurrences more) were identified as being well-learned chunks of the experimental sequence. Chance frequency was determined in the following way: For each letter, the probability of being the first letter of a chunk was 25 , and the probability of being one of the following letters was 33 (since, with immediate repetitions being prohibited, only one of three different letters could be selected). For any chunk, the chance level of occurrence was computed as the product of the individual probabilities of each chunk element and the number of times a chunk of this length could occur in a 160 -item block. For a chunk of three elements, for example, this formula would yield a chance level of $.25 \times .33 \times .33 \times 158=4.3$ occurrences.

EEG was averaged off line for epochs of $800 \mathrm{msec}$, starting $100 \mathrm{msec}$ prior to stimulus onset and ending $700 \mathrm{msec}$ afterwards. Trials with eyeblinks (indicated by a voltage recorded at $\mathrm{FPz}$ exceeding $\pm 50 \mu \mathrm{V}$ ), and overt response errors were excluded from analysis. EEG was averaged separately for deviant letters, letters immediately following the deviant, and the remaining standard letters. Only the ERPs to deviant letters and to standard letters that were not immediately preceded by a deviant letter were further analyzed. In order to obtain a sufficient number of epochs for each average waveform, the factors half (first vs. second experimental half) and chunk (chunk-internal vs. chunk-external items) were analyzed separately. Thus, on one hand, separate averages for standard and deviant letters were computed for each experimental half (Blocks 1-9 vs. Blocks 10-18, averaged over chunk-internal and chunk-external positions), and, on the other hand, separate averages for standard and deviant letters were computed for the parts of the sequence that were identified as chunks and for the remaining parts of the sequence (chunk-internal vs. chunk-external positions, averaged over both experimental halves). To test whether the difference between standard and deviant letters had an effect on the ERP waveforms, repeated measures analyses of variance (ANOVAs) were performed for $\mathrm{N} 2 \mathrm{~b}$ and $\mathrm{P} 3 \mathrm{~b}$ amplitudes for the following variables: stimulus type (standard vs. deviant), electrode ( $\mathrm{FPz}, \mathrm{Fz}, \mathrm{Cz}, \mathrm{Pz}, \mathrm{C3}^{\prime}, \mathrm{C4}^{\prime}$ ), and experimental half (Blocks $1-9$ vs. Blocks $10-18)$ on the one hand, and stimulus type, electrode, and chunk (chunk-internal vs. chunk-external) on the other hand. N2b amplitude was determined as the mean amplitude within the 230-330 msec poststimulus time window; $\mathrm{P} 3 \mathrm{~b}$ amplitude was determined as the mean amplitude within the $400-600$ msec poststimulus time window.

Mean RTs were determined separately for deviant letters, for standard letters that were immediately preceded by a deviant stimulus, and for the remaining standard letters. Standard letters immediately preceded by a deviant item were excluded from further analysis. For the remaining RT data, a repeated measures ANOVA was performed for the factors experimental half (first vs. second experimental half), stimulus type (standard vs. deviant), and chunk (chunk-internal vs. chunk-external).

\section{Results}

\section{Postexperimental Measures}

Verbal reports. One participant was unable to verbally reproduce any part of the sequence. Reproduction performance of the remaining participants ranged from 3 to 8 successive elements of the sequence. Seven participants were able to identify the experimental sequence presented on a sheet of paper.

Production tasks. Figure 1 gives an overview of the mean number of sequence parts with three or more elements reproduced more than once and with more than chance frequency in the inclusion and exclusion block, respectively. Additionally, the mean number of chunks (i.e., sequence parts that were produced in the inclusion block with more than chance frequency and substantially more often than in the exclusion block) is depicted. Analysis of the reproduction tasks revealed that the length of well-learned chunks ranged from 3 to 8 elements, with a mean chunk length of 4.5 elements. At least one chunk was well learned by every participant, and no participant 


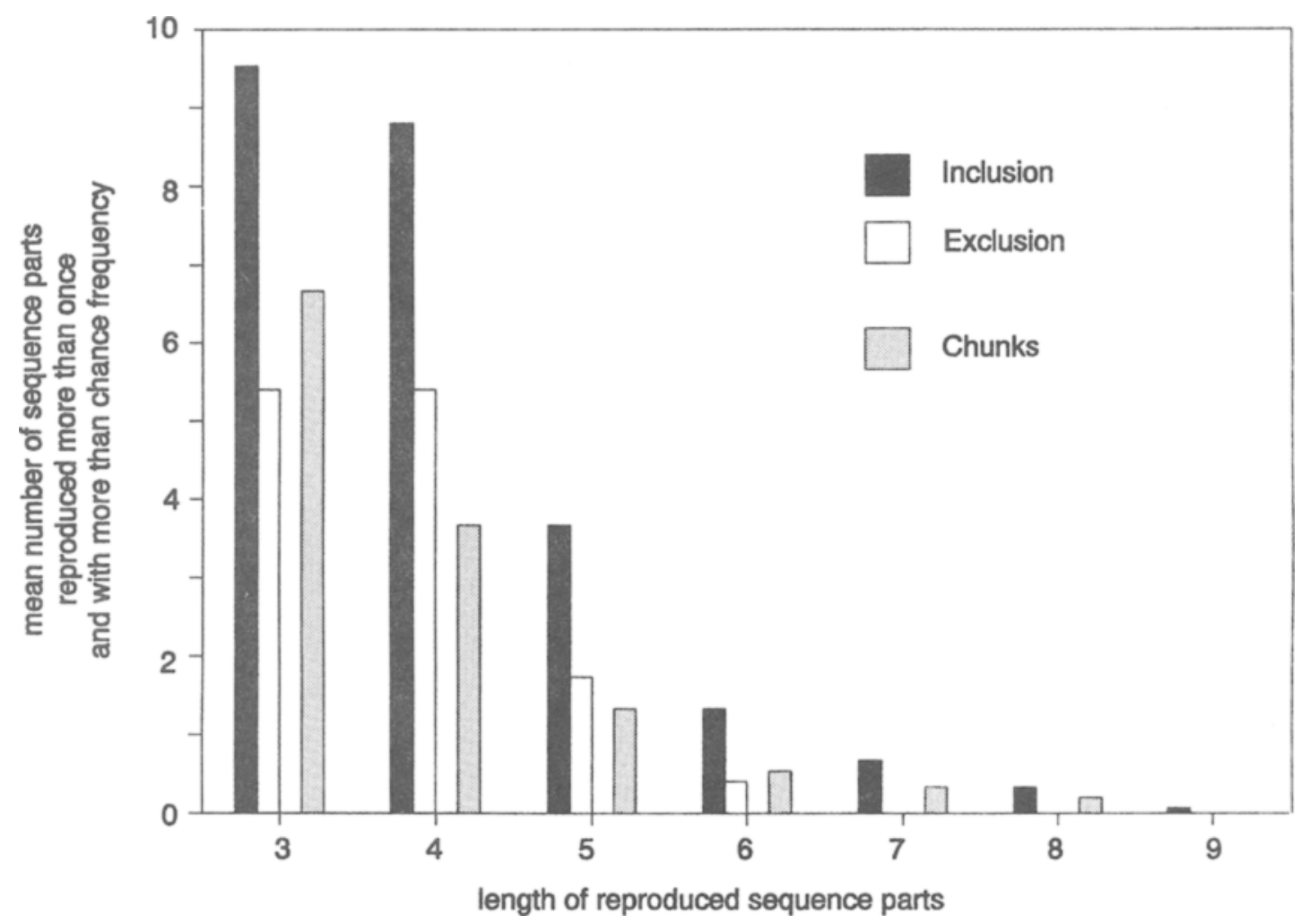

Figure 1. Results of reproduction tasks. Black and white bars represent the mean number of sequence parts produced more than once and with more than chance frequency in the inclusion block (black bars) and in the exclusion block (white bars). Gray bars represent the mean number of sequence parts identified as "well-learned chunks" (i.e., those sequence parts that were produced substantially more often in the inclusion condition than in the exclusion condition; for details, see text). Results are plotted separately for chunks of different lengths, irrespective of chunk identity (note that, for any given length, 16 different chunks are possible).

acquired more than three chunks of the experimental sequence (average $=1.8$ chunks). That is, on average, there were about 8 elements of the 16-item sequence identified as chunk-internal items.

Behavioral performance (See Figure 2). In the first experimental half, the overall RT was $585 \mathrm{msec}(S D=$ $83 \mathrm{msec})$ for standard letters and $654 \mathrm{msec}(S D=$ $88 \mathrm{msec}$ ) for deviant letters at chunk-internal positions, and the overall RT was $597 \mathrm{msec}(S D=79 \mathrm{msec})$ for standard letters and $658 \mathrm{msec}(S D=63 \mathrm{msec})$ for deviant letters at chunk-external positions (see Figure 2). In the second experimental half, the overall RT was $514 \mathrm{msec}(S D=79 \mathrm{msec})$ for standard letters and $605 \mathrm{msec}(S D=75 \mathrm{msec})$ for deviant letters at chunkinternal positions, and the overall RT was $528 \mathrm{msec}$ $(S D=72 \mathrm{msec})$ for standard letters and $618 \mathrm{msec}(S D=$ $60 \mathrm{msec}$ ) for deviant letters at chunk-external positions (Figure 2). The RT difference between standard and deviant stimuli was reflected in a main effect of stimulus type $\left[F(1,14)=63.88, M S_{\mathrm{e}}<0.01, p<.001\right]$, whereas the main effect of chunk failed to reach significance $\left[F(1,14)=3.13, M S_{\mathrm{e}}<0.01, p<.098\right]$.
A main effect of experimental half $[F(1,14)=68.41$, $\left.M S_{\mathrm{e}}<0.01, p<.001\right]$ indicated that responses became faster during the course of the experiment. Inspection of Figure 2 (upper panel) suggests that learning took place mainly during the first part of the experiment, in which RTs to standard items became increasingly faster. ${ }^{1}$ Consequently, the overall effect of stimulus type was larger in the second experimental half than in the first (see Figure 2 , lower panel). This was supported by a two-way interaction of experimental half $\times$ stimulus type $[F(1,14)=$ 9.15, $\left.M S_{\mathrm{e}}<0.01, p<.009\right]$. Most interestingly, none of the interactions containing the factor chunk reached significance (all $F \mathrm{~s}<1.2$; see Figure 2, lower panel).

\section{Electrophysiological Measures}

N2b amplitude. Although Figure 3 shows that the mean amplitude in the $\mathrm{N} 2 \mathrm{~b}$ range was more negative for deviant stimuli than for standard stimuli, ${ }^{2}$ there was neither a main effect of stimulus type nor a main effect of experimental half in this latency range (both $F \mathrm{~s}<2.6$ ). However, the main effect of chunk and the interaction between stimulus type and chunk approached significance 

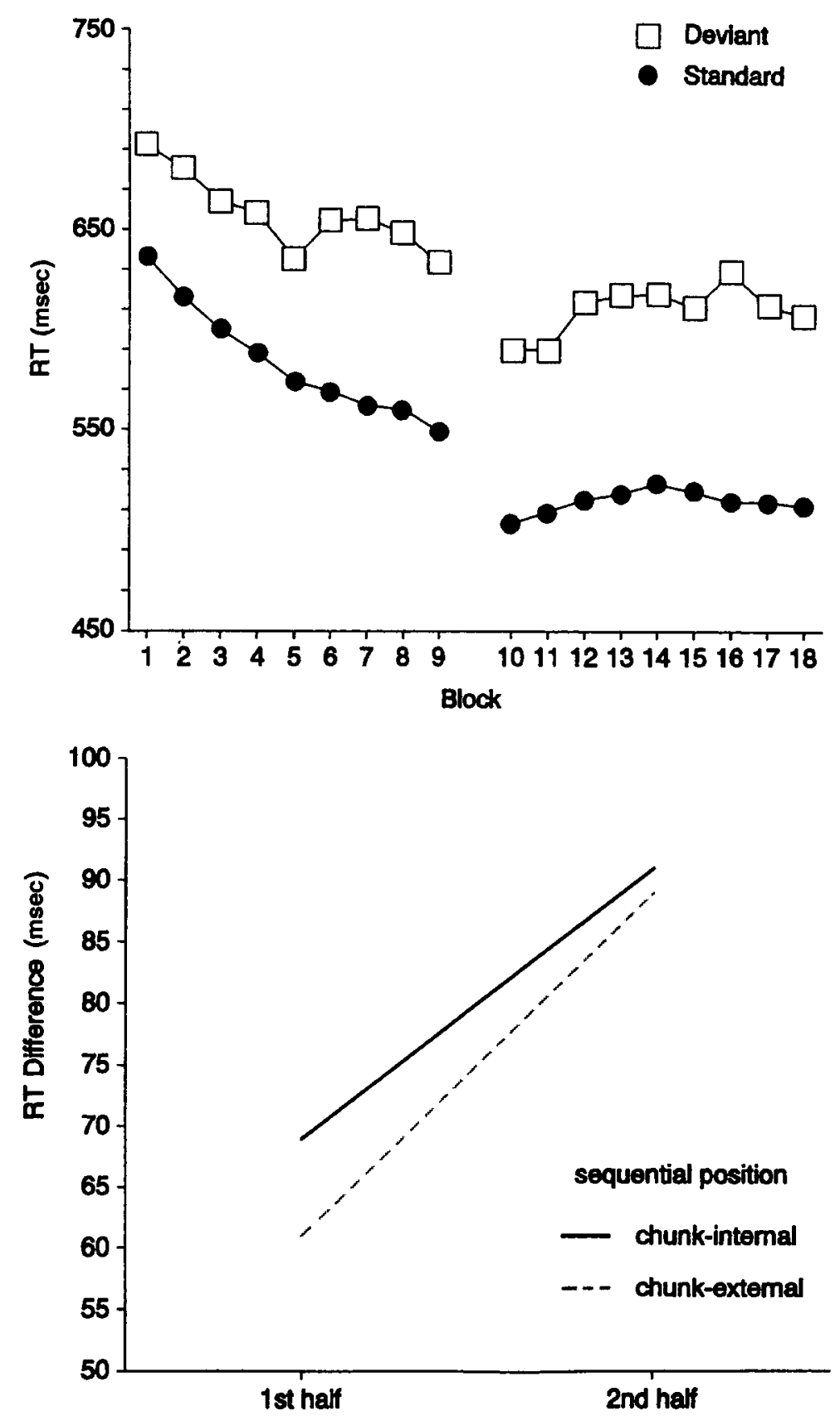

Figure 2. Upper panel: Mean RT (in milliseconds) for correct responses in each of the 18 experimental blocks. Black circles represent RTs to standard stimuli; white squares represent RTs to deviant stimuli. Lower panel: Effect of stimulus deviance on RT (standard RT - deviant $R T$ ) in the first and second experimental halves, plotted separately for chunk-internal stimuli (solid line) and chunk-external stimuli (dashed line).

$\left[F(1,14)=3.87, M S_{\mathrm{e}}=0.54, p<.069 ;\right.$ and $F(1,14)=$ $4.12, M S_{\mathrm{e}}=0.40, p<.062$, respectively]. Figure 3 shows that only chunk-internal deviants elicited an enlarged N2b component. For chunk-external deviants, no such tendency was visible. This observation was supported by additional ANOVAs, conducted for chunk-internal and chunk-external positions separately: At chunk-internal positions, there was a significant effect of stimulus type $\left[F(1,14)=5.34, M S_{\mathrm{e}}=0.48, p<.037\right]$, which was missing at chunk-external positions $[F(1,14)=0.02]$. In order to test whether this effect should be regarded as a "real" $\mathrm{N} 2 \mathrm{~b}$ effect or whether it could be due to the shift in P3b 


\section{chunk-internal}
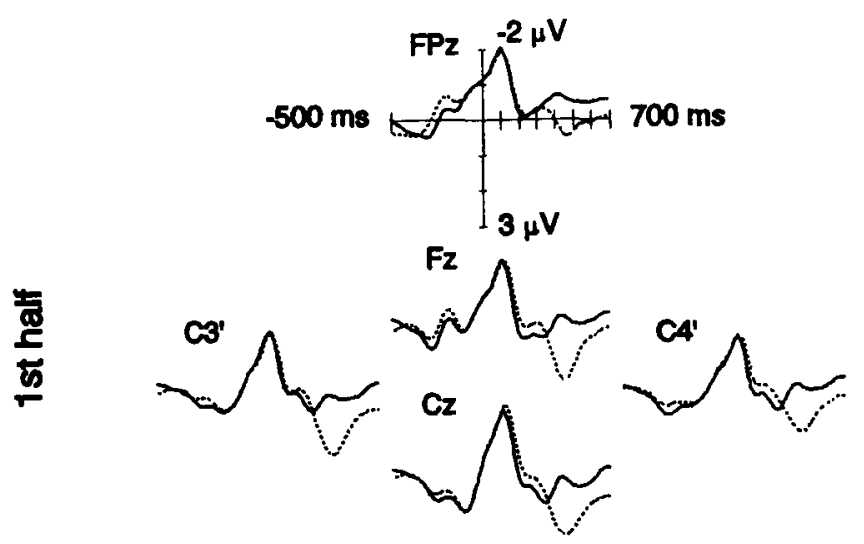<smiles>CCCCCCCCCCCC</smiles>

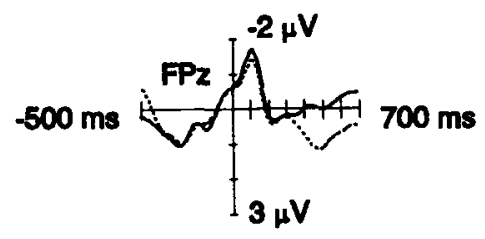

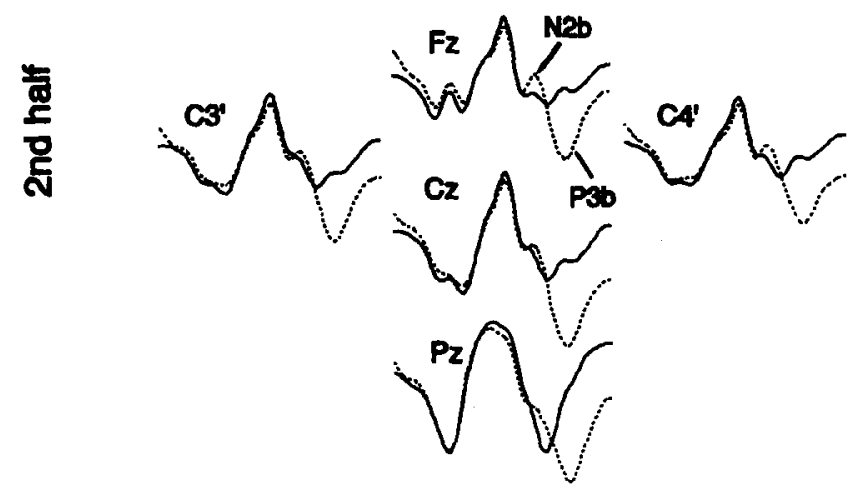

\section{chunk-external}

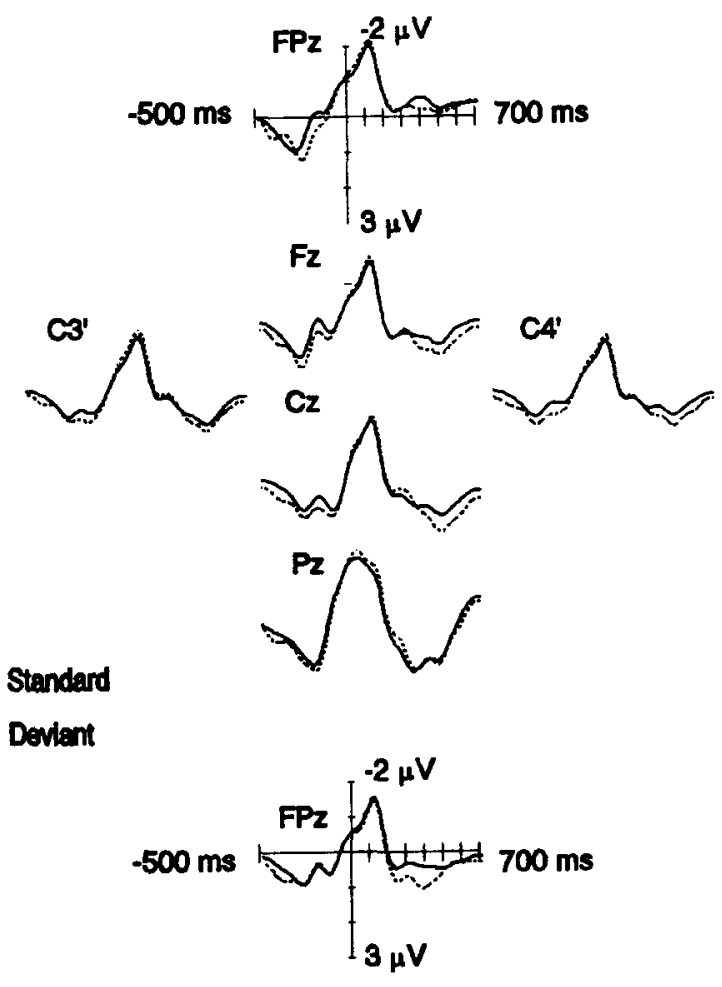

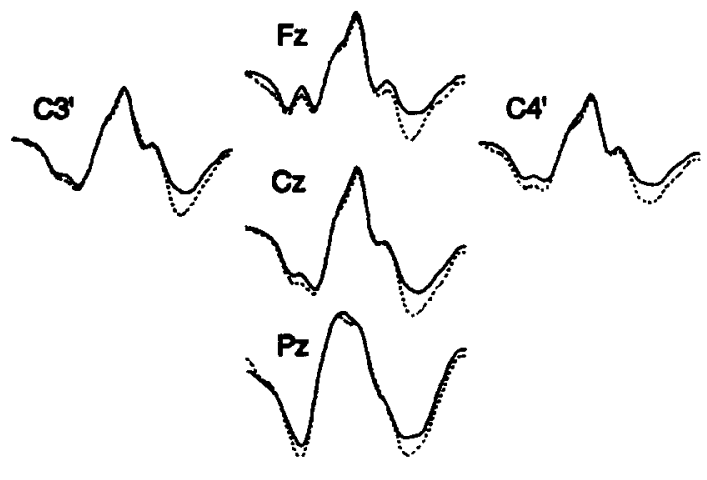

Figure 3. Grand mean average ERP waveforms for standard items (solid line) and deviant items (dashed line), plotted separately for each experimental half and for chunk-internal and chunk-external positions. Note that negative voltage is plotted upward. These waveforms were filtered with a $20-\mathrm{Hz}$ low-pass filter (statistical analysis, however, was conducted on unfiltered averages).

onset elicited by chunk-internal deviants at $\mathrm{Pz}$ (see Figure 3 ), the analysis was repeated including all electrode sites except $\mathrm{Pz}$. The result was clear-cut: There was still a significant effect of stimulus type at chunk-internal positions and no such effect at chunk-external positions $[F(1,14)=6.11, p<.027$; and $F(1,14)=0.2$, respectively $]$.

P3b amplitude. The mean amplitude in the $\mathrm{P} 3 \mathrm{~b}$ range was more positive for deviant stimuli than for standard stimuli, yielding a significant main effect of stimulus type in this latency range $\left[F(1,14)=42.67, M S_{\mathrm{e}}=1.47, p<\right.$ $.001]$. Moreover, there was a significant main effect of ex- perimental half on $\mathrm{P} 3 \mathrm{~b}$ amplitude $\left[F(1,14)=8.96, M S_{\mathrm{e}}=\right.$ $0.49, p<.01]$, and an interaction between stimulus type and experimental half $\left[F(1,14)=8.96, M S_{\mathrm{e}}=0.29, p<\right.$ $.01]$, reflecting a larger $\mathrm{P} 3 \mathrm{~b}$ effect in the second experimental half than in the first half.

Additionally, there was a main effect of chunk on $\mathrm{P} 3 \mathrm{~b}$ amplitude $\left[F(1,14)=6.75, M S_{\mathrm{e}}=0.56, p<.021\right]$, together with an interaction between stimulus type and chunk $\left[F(1,14)=11.73, M S_{\mathrm{e}}=2.17, p<.004\right]$, with the P3b effect elicited by deviant stimuli being large at chunkinternal and much smaller at chunk-external positions. 
Additional repeated measures ANOVAs, conducted separately for chunk-internal and chunk-external positions, revealed an effect of stimulus type on P3b amplitude only at chunk-internal positions $\left[F(1,14)=30.03, M S_{\mathrm{e}}=2.72\right.$, $p<.001]$. Although, at chunk-external positions the $\mathrm{P} 3 \mathrm{~b}-$ effect was almost significant $\left[F(1,14)=3.91, M S_{\mathrm{e}}=0.92\right.$, $p<.068$ ], planned pairwise comparisons in form of paired $t$ tests revealed no significant effect of stimulus type at any single electrode location.

\section{Discussion}

In two previous studies (Baldwin \& Kutas, 1997; Eimer et al., 1996), it was found that in incidental learning paradigms, deviant events infrequently interspersed in a context of regular events elicit a characteristic pattern of enlarged N2b/P3b components. These effects were largest for participants showing substantial explicit knowledge about the underlying regularity, although, even under conditions in which participants had not acquired substantial explicit knowledge, some N2b/P3b effects occurred. The objective of the present experiment was to investigate whether these effects are at least partly due to the existence of consciously accessible knowledge of welllearned sequence parts. It was assumed that, in an incidental learning situation in which participants are confronted with a repeating event sequence, the acquisition of explicit knowledge may start by first recognizing the repetition of one or two small parts of the sequence (chunks) and then proceeds by adding more and more elements to the initial chunk.

In order to encourage chunk learning, a 16-item sequence with an arbitrary stimulus-response mapping was employed. No participant was able to reproduce the complete sequence, but each participant showed explicit knowledge about at least small chunks, as indicated by the results of the process dissociation procedure in the two reproduction blocks. This procedure established a measurement of explicit knowledge independently from RT effects. Note, however, that it was not employed as a measure of implicit knowledge: Since there is as yet no generally accepted method of estimating the proportion of implicit influences on task performance (e.g., Reingold \& Toth, 1996), the present experiment focused on explicitly learned chunks only.

It was assumed that if incidental learning relies on the acquisition of sequence parts, ERP effects obtained for well-learned chunks should differ markedly from those obtained for the remaining sequence positions: At chunkinternal positions, but not at chunk-external positions, deviant items were expected to give rise to N2b/P3b effects similar to those observed for participants exhibiting explicit knowledge in previous studies (Baldwin \& Kutas, 1997; Eimer et al., 1996). This hypothesis was confirmed. As shown in Figure 3, N2b effects and P3b effects were elicited by deviant stimuli at chunk-internal positions. In contrast, both effects were substantially reduced at chunkexternal positions. It has to be noted, though, that the in- teraction of chunk and stimulus type in the N2b latency range only approached statistical significance and that there was an almost significant P3b effect at chunkexternal positions, probably due to a significant effect at these positions in the second experimental half (see Figure 3). However, additional analyses revealed that reliable effects of stimulus type on the $\mathrm{N} 2 \mathrm{~b}$ and $\mathrm{P} 3 \mathrm{~b}$ components were elicited only at chunk-internal positions. Since these effects were obtained in a within-subjects design, the hypothesis is further supported that these ERP indicators can be used as an on-line measurement of explicit knowledge in addition to backward inferences drawn from memory tests applied after training.

On the other hand, the effect of stimulus deviance on RTs did not differ between chunk-external and chunkinternal positions. Although RTs tended to be slightly faster at chunk-internal positions than at chunk-external positions ( $11 \mathrm{msec}$ on average), the difference between RT to standard items and RT to deviant items was about the same in both cases ( 80 and $75 \mathrm{msec}$, respectively). Thus, one may conclude that although in the present study deviant stimuli elicited longer RTs, an enhanced N2b deflection, and an enlarged P $3 \mathrm{~b}$ component, these effects seem to be differentially sensitive to implicitly and explicitly represented knowledge: While $\mathrm{P} 3 \mathrm{~b}$ and $\mathrm{N} 2 \mathrm{~b}$ effects were mainly restricted to chunk-internal positions, RT effects turned out to be nearly independent of chunk learning.

This dissociation of behavioral and electrophysiological measures stands in marked contrast to the findings reported in Baldwin and Kutas (1997), in which RT effects and ERP effects were closely related. However, there are rather substantial differences in experimental design that may render a direct comparison of the results of these studies impossible. First, in the Baldwin and Kutas study, an artificial finite-state grammar was employed governing the regularities of stimulus presentation, whereas in the present experiment, a continuously repeating 16-item sequence of four different stimuli was delivered. Second, in the Baldwin and Kutas study, explicit knowledge was induced by teaching the participants the rules of the underlying grammar prior to the serial RT task, whereas in the present experiment, learning of the stimulus sequence was incidental. Third, and perhaps most important, in the Baldwin and Kutas study, participants were required to respond only on those trials on which the target had performed a certain predefined movement, whereas in the present experiment, a response had to be given on each trial. Taken together, these differences in experimental design may have led participants to adopt completely different strategies in dealing with the task at hand. Note, for example, that in the present experiment, but not in the Baldwin and Kutas study, the repeating stimulus sequence was accompanied by a correspondingly repeating sequence of manual responses. Thus, one may argue that in the present experiment, motor learning may have played an important role in sequence 
acquisition, whereas in the Baldwin and Kutas study, motor learning was largely irrelevant for acquiring knowledge about the underlying grammar (the role of motor learning in sequence acquisition is still under discussion; e.g., see Cohen et al., 1990; Eimer et al., 1996; Fendrich, Healy, \& Bourne, 1991; Zießler, 1994).

Recent findings have shown that motor learning may be mediated by subcortical structures, such as the basal ganglia, which form a closed field not recordable from scalp sites (e.g., Grafton et al., 1992; Jenkins, Brooks, Nixon, Frackowiak, \& Passingham, 1994). Thus, if one assumes that motor learning played a major role for sequence acquisition in the present experiment, the dissociation between substantial RT effects and missing ERP effects of stimulus deviance at chunk-external positions could be explained as follows: While the RT effects at chunk-external positions provide evidence that the participants acquired some knowledge about parts of the sequence they were not able to reproduce consciously at the end of the experiment, the failure to find reliable $\mathrm{N} 2 \mathrm{~b} / \mathrm{P} 3 \mathrm{~b}$ effects at these positions indicates that this knowledge is based mainly on processes of motor learning, which operate at subcortical brain structures and are not recordable with the EEG methods employed in the present experiment. This is not to say, however, that motor learning is identical to implicit learning in the present task or that purely implicit learning took place at chunk-external positions. As already mentioned, there exists as yet no generally accepted criterion to unequivocally identify implicit knowledge (Reingold \& Toth, 1996). That is, even if the assumption is correct that RT effects at chunkexternal positions are due to motor learning processes, it is still unclear from the present data whether these processes are implicit or explicit. Thus, one may conclude that RT effects generally represent a mixture of explicitly and implicitly represented knowledge about the sequential structure, whereas $\mathrm{N} 2 \mathrm{~b}$ amplitude and $\mathrm{P} 3 \mathrm{~b}$ amplitude more purely indicate the amount of consciously available knowledge. ${ }^{3}$

Moreover, although one may speculate on the basis of the dissociation between RT effects and ERP effects that there are indeed substantial differences between explicit and implicit knowledge, it remains an open question whether these differences indicate that explicit and implicit knowledge are represented by different memory systems. It seems suggestive to propose that one memory system (implicit, procedural) is exclusively linked to motor control, while another system (explicit, declarative) is linked to both motor control and conscious performance. However, the possibility remains that there is only one common mechanism. One could assume, for example, that implicit and explicit representations differ in their representational strength: For the parts of the sequence that are already well learned, a transfer to a new task (i.e., reproduction) can take place, whereas for the parts that are not well learned as yet, learning effects are obtained only within the practiced task itself. According to this view, the dissociation between RT effects and ERP effects reflects the existence of different processing stages within a continuum of gradually increasing representational strength. On the basis of the present results, a combination of electrophysiological measures and process dissociation techniques seems to be a promising approach to investigate more closely the issue of whether one common or two separate mechanisms underlie sequence learning.

\section{REFERENCES}

Baldwin, K. B., \& Kutas, M. (1997). An ERP analysis of implicit structured sequence learning. Psychophysiology, 34, 74-86.

Cohen, A., Ivry, R. I., \& KeELE, S. W. (1990). Attention and structure in sequence learning. Journal of Experimental Psychology: Learning, Memory, \& Cognition, 16, 17-30.

Curran, T., \& Keele, S. W. (1993). Attentional and nonattentional forms of sequence learning. Journal of Experimental Psychology: Learning, Memory, \& Cognition, 19, 189-202

Donchin, E. (1981). Surprise ... Surprise? Psychophysiology, 18, 493-513.

Eimer, M., Goschke, T., Schlaghecken, F., \& Stürmer, B. (1996). Explicit and implicit learning of event sequences: Evidence from event-related brain potentials. Journal of Experimental Psychology: Learning, Memory, \& Cognition, 22, 970-987.

Fendrich, D. W., Healy, A. F., \& Bourne, L. E. (1991). Long-term repetition effects for motoric and perceptual procedures. Journal of $E x-$ perimental Psychology: Learning, Memory, \& Cognition, 17, 137-151.

GaRdinER, J. M., \& JAVA, R. I. ( 1993). Recognising and remembering. In A. Collins, M. A. Conway, S. E. Gathercole, \& P. E. Morris (Eds.), Theories of mind (pp. 163-188). Hillsdale, NJ: Erlbaum.

Grafton, S. T., Mazziotta, J. C., Presty, S., Friston, K. J., Frackowiak, R. S. J., \& PhElPS, M. E. (1992). Functional anatomy of human procedural learning determined with regional cerebral blood flow and PET. Journal of Neuroscience, 12, 2542-2548.

HarTMAn, M., KNOPMAN, D. S., \& NisSEN, M. J. (1989). Implicit learning of new verbal associations. Journal of Experimental Psychology: Learning, Memory, \& Cognition, 15, 1070-1082.

JACOBY, L. L. (1991). A process dissociation framework: Separating automatic from intentional uses of memory. Journal of Memory \& Language, 30, 513-541

Jenkins, I. H., Brooks, D. J., Nixon, P. D., Frackowiak, R. S. J., \& Passingham, R. E. (1994). Motor sequence learning: A study with positron emission tomography. Journal of Neuroscience, 14, 3775-3790.

Joordans, S., \& MERIKLE, P. M. (1993). Independence or redundancy? Two models of conscious and unconscious influences. Journal of Experimental Psychology: General, 4, 462-467.

LeWICKI, P., Hill, T., \& Bizot, E. (1988). Acquisition of procedural knowledge about a pattern of stimuli that cannot be articulated. Cognitive Psychology, 20, 24-37.

NisSEN, M. J., \& Bullemer, P. (1987). Attentional requirements of learning: Evidence from performance measures. Cognitive Psychology, 19, 1-32.

Nissen, M. J., Willingham, D., \& Hartman, M. (1989). Explicit and implicit remembering: When is learning preserved in amnesia? Neuropsychologia, 27, 341-352.

Perruchet, P. (1994). Learning from complex rule-governed environments: On the proper functions of nonconscious and conscious processes. In C. Umiltà \& M. Moscovitch (Eds.), Attention and performance $X V$ : Conscious and nonconscious information processing (pp. 811-835). Cambridge, MA: MIT Press.

Perruchet, P., \& Amorim, M. A. (1992). Conscious knowledge and changes in performance in sequence learning: Evidence against dissociation. Journal of Experimental Psychology: Learning, Memory, \& Cognition, 18, 785-800 
Picton, T. W., \& Hillyard, S. A. (1988). Endogenous event-related potentials. In T. W. Picton (Ed.), Human event-related potentials: EEG handbook (rev. series, Vol. 3, pp. 361-426). Amsterdam: Elsevier.

REBER, A. S. (1989). Implicit learning and tacit knowledge. Journal of Experimental Psychology: General, 118, 219-235.

REED, J., \& JOHNSON, P. (1994). Assessing implicit learning with indirect tests: Determining what is learned about sequence structure. Journal of Experimental Psychology: Learning, Memory, \& Cognition, 20, 585-594.

Reingold, E. M., \& Toth, J. P. (1996). Process dissociation versus task dissociation: A controversy in progress. In G. Underwood (Ed.), Implicit cognition (pp. 159-202). Oxford: Oxford University Press.

RitTer, W., Ford, J. M., Gaillard, A. K. W., HarTer, M. R., Kutas, M., NäÄtänen, R., Polich, J., Renault, B., \& Rohrbaugh, J. (1984). Cognition and event-related potentials: 1 . The relationship of negative potentials and cognitive processes. In R. Karrer, J. Cohen, \& P. Tueting (Eds.), Brain and information: Event-related potentials (Annals of the New York Academy of Sciences, Vol. 425, pp. 24-38). New York: New York Academy of Sciences.

RoEDiger, H. L., III (1990a). Implicit memory: A commentary. Bulletin of the Psychonomic Society, 28, 373-380.

ROEDIGER, H. L., III (1990b). Implicit memory: Retention without remembering. American Psychologist, 45, 1043-1056.

Ruchkin, D. S., Sutton, S., \& Tueting, P. (1975). Emitted and evoked P300 potentials and variation in stimulus probability. Psychophysiology, 12, 591-595.

SCHACTeR, D. L. (1994). Priming and multiple memory systems: Perceptual mechanisms of implicit memory. In D. L. Schacter \& E. Tulving (Eds.), Memory systems 1994 (pp. 233-268). Cambridge, MA: MIT Press.

SEGER, C. A. (1994). Implicit learning. Psychological Bulletin, 115, 163-196.

Shanks, D. R., Green, R. E. A., \& Kolodny, J. (1994). A critical examination of the evidence for unconscious (implicit) learning. In C. Umiltà \& M. Moscovitch (Eds.), Attention and performance XV. Conscious and nonconscious information processing (pp. 837-860). Cambridge, MA: MIT Press.

ShanKs, D. R., \& St. JoHN, M. F. (1994). Characteristics of dissociable human learning systems. Behavioral \& Brain Sciences, 17, 367-447.

SQuIRE, L. (1994). Declarative and nondeclarative memory: Multiple brain systems supporting learning and memory. In D. L. Schacter \& E. Tulving (Eds.), Memory systems 1994 (pp. 203-232). Cambridge, MA: MIT Press.
Souires, K. C., Donchin, E., Herning, R. I., \& McCarthy, G. (1977) On the influence of task relevance and stimulus probability on eventrelated-potential components. Electroencephalography \& Clinical Neurophysiology, 42, 1-14.

Squires, K. C., Wickens, C., Squires, N. K., \& Donchin, E. (1976). The effect of stimulus sequence on the waveform of the cortical eventrelated potential. Science, 193, 1142-1146.

StADLER, M. A. (1989). On learning complex procedural knowledge. Journal of Experimental Psychology: Learning, Memory, \& Cognition, 15, 1061-1069.

StADLER, M. A. (1993). Implicit serial learning: Questions inspired by Hebb (1961). Memory \& Cognition, 21, 819-827.

Willingham, D. B., Greeley, T., \& Bardone, A. M. (1993). Dissociation in a serial response time task using a recognition measure: Comment on Perruchet and Amorim (1992). Journal of Experimental Psychology: Learning, Memory, \& Cognition, 19, 1424-1430.

Willingham, D. B., Nissen, M. J., \& Bullemer, P. (1989). On the development of procedural knowledge. Journal of Experimental Psychology: Learning, Memory, \& Cognition, 15, 1047-1060.

ZIERLER, M. (1994). The impact of motor responses on serial-pattern learning. Psychological Research, 57, 30-41.

\section{NOTES}

1. Note that the RT difference between standard trials and deviant trials in the first block indicates that learning already began during the first 16 repetitions of the sequence. Similar findings of an early onset of deviance effects on RTs are reported in Baldwin and Kutas (1997) and in Eimer et al. (1996).

2. The positive-going deflections visible at all electrode sites prior to stimulus onset represent response-related components of the ERP. They survived the averaging procedure because the next stimulus always appeared with a constant interval of $200 \mathrm{msec}$, after response execution for the previous trial. Note, however, that there is virtually no difference between ERPs for standard and deviant items prior to stimulus onset.

3. One may even speculate that the $P 3 \mathrm{~b}$ effect visible at chunk-external positions in the second experimental half represents the onset of explicit knowledge developing even for these positions. Unfortunately, there was no way to test this hypothesis on the basis of the present data.

(Manuscript received January 27, 1998; revision accepted for publication July 5, 1999.) 